\title{
PHOX2B expression in bone marrow and peripheral blood is associated with metastasis and prognosis in patients with neuroblastoma
}

\section{Hongjun Fan}

Beijing Children's Hospital https://orcid.org/0000-0002-1034-3221

Tianyu Xing

Beijing Children's Hospital

Huimin Hong

Beijing Children's Hospital

Chao Duan

Beijing Children's Hospital

\section{Wen Zhao}

Beijing Children's Hospital

Qian Zhao

Beijing Children's Hospital

\section{Xisi Wang}

Beijing Children's Hospital

Cheng Huang

Beijing Children's Hospital

\section{Shuai Zhu}

Beijing Children's Hospital

Mei Jin

Beijing Children's Hospital

\section{Yan Su}

Beijing Children's Hospital

Chao Gao

Beijing Children's Hospital

Xiaoli Ma ( $\sim$ mxl1123@vip.sina.com )

https://orcid.org/0000-0001-7930-4944

Research article

Keywords: Neuroblastoma, PHOX2B, Molecular marker, Metastasis, Prognosis 
Posted Date: March 10th, 2020

DOI: https://doi.org/10.21203/rs.3.rs-16678/v1

License: (c) (i) This work is licensed under a Creative Commons Attribution 4.0 International License. Read Full License 


\section{Abstract}

Background Paired-like homeobox 2B (PHOX2B) is specifically expressed in the nervous system including neuroblastoma cells, but little is known about the clinical significance of the expression of PHOX2B in bone marrow (BM) and peripheral blood (PB) samples of newly diagnosed neuroblastoma patients. Methods The expression of PHOX2B in 276 paired BM and PB samples of neuroblastoma patients at diagnosis was tested by quantitative reverse transcriptase polymerase chain reaction (RT-PCR). Then the relationship between $\mathrm{PHOX}$ 2B level and clinical characteristics including metastasis and prognosis was explored by receiver operating characteristic (ROC) analysis and Kaplan-Meier method. Results We identified the combined expression of PHOX2B in both BM and PB provided a high diagnostic accuracy for metastasis of neuroblastoma patients (AUC $=0.920$ ) with the sensitivity and specificity of $86.7 \%$ and $92.9 \%$, respectively. At last, 246 patients were enrolled for prognostic analysis. The median follow-up time was 22 months. Positive expressions of PHOX2B in BM and PB at diagnosis were associated with worse EFS and OS in neuroblastoma patients $(P<0.05)$. What's worse, $19.7 \%(31 / 157)$ and $6.4 \%(10 / 157)$ patients with positive expression of $\mathrm{PHOX} 2 \mathrm{~B}$ in $\mathrm{BM}$ and $\mathrm{PB}$ samples in low/intermediate-risk group also had shorter EFS and poor OS $(\mathrm{P}<0.05)$. CONCLUSIONS The expressions of PHOX2B in BM and PB were high in patients with unfavorable clinical characteristics. PHOX2B could be an appropriate biomarker for predicting metastasis and prognosis in patients with neuroblastoma.

\section{Background}

Neuroblastoma, originating from the peripheral sympathetic nervous system, accounts for $12 \%$ of deaths associated with cancer in children [1]. Although patients with low- and intermediate-risk neuroblastoma have favorable outcomes, the prognosis of high-risk patients is still dismal with 5-year overall survival (OS) less than $50 \%$ [2]. Nearly one-half of neuroblastoma patients are classified into the high-risk group with metastasis at the initial diagnosis [3]. Positron emission tomography-computed tomography (PET$\mathrm{CT}$ ), computed tomography (CT), magnetic resonance imaging (MRI) and iodine-123

metaiodobenzylguanidine ( ${ }^{123} \mathrm{I}-\mathrm{MIBG}$ ) scanning are widely used in detecting neuroblastoma patients with distant metastasis. However, repeated CT or ${ }^{123}$ I-MIBG scans during the long-term treatment expose children to radiation and also unable to detect small primary lesions and metastases accurately. Therefore, new methods should be tested for predicting metastasis in neuroblastoma patients.

Liquid biopsy, including circulating tumor cells (CTCs), cell-free tumor DNAs (ctDNAs), as well as RNAs, proteins, and exosomes, has been introduced as a new method for the diagnosis of cancer metastasis in the past decades $[4,5]$. Since the detection of neuroblastoma cells in the blood of patients with disseminated disease by Moss et al in the 1990s [6], some studies have been devoted to the development and improvement of the methods for liquid biopsy in neuroblastoma patients. Paired-like homeobox 2B (PHOX2B) gene, located on chromosome 4p13 [7], is specifically expressed in the nervous system including neuroblastoma cells, but not in normal bone marrow or peripheral blood cells of patients $[8,9]$. Previously, some studies showed PHOX2B could be used as a biomarker for minimal residual disease (MRD) and predicting prognosis in neuroblastoma patients [9-12]. In our study, we evaluated the 
diagnostic significance of PHOX2B expression in bone marrow (BM) and peripheral blood (PB) for disseminated diseases and patients' outcomes.

\section{Methods}

\section{Patients and samples}

Paired BM and PB samples were collected from 276 newly diagnosed neuroblastoma patients, who were enrolled in our center from June 1st, 2017 to December 31th, 2018. The study was approved by the Ethical Committee of our Hospital. Informed written consent was obtained from each participant according to the Declaration of Helsinki. The International Neuroblastoma Risk Group Staging System (INRGSS) was used for stratifying patients with different clinical stages at the time of diagnosis before any treatment [13]. We defined cases as patients with metastasis by the evaluation of PET-CT, bone scans, CT, MRI, bone marrow aspirates and trephine biopsies at diagnosis [1]. Each patient had two sites bone marrow aspirates of the sternum and ilium at diagnosis. The patients were treated in different risk groups according to prognostic category $[2,14]$, and the chemotherapy regimen was guided by $\mathrm{BCH}-$ 2007-NB $[15,16]$.

\section{Real-time Polymerase Chain Reaction}

The Trizol Reagent was used to extract total RNA from $2 \mathrm{~mL} \mathrm{BM}$ and PB samples, and then the RNA was reverse transcribed into cDNA [17]. RT-PCR was performed in a ViiA 7 Dx System (Applied Biosystems, Carlsbad, USA). The expression of PHOX2B gene was detected by the Applied Biosystems ${ }^{\mathrm{TM}}$ TaqMan $^{\mathrm{TM}}$ Array Human Hox Genes (Cat.No 4414103, Applied Biosystems, Foster city, CA). Reactions were carried out in $12.5 \mu \mathrm{L}\left(6.4 \mu \mathrm{L} \mathrm{H} \mathrm{H}_{2} \mathrm{O}, 1 \mu \mathrm{L}\right.$ forward and reverse primers, $1 \mu \mathrm{L}$ cDNA (input $80 \mathrm{ng}$ ), $6.25 \mu \mathrm{L}$ Mastermix, $0.75 \mu \mathrm{L}$ PHOX2B Assay), started with 10 minutes at $95^{\circ} \mathrm{C}$ followed by 50 cycles $\left(15\right.$ seconds at $95^{\circ} \mathrm{C}$ followed by 1 minute at $60^{\circ} \mathrm{C}$ ). All RT-PCR experiments were performed in triplicate, and mean values were used. The housekeeping gene GUS was used as the gene for normalization. The quantity of the expression of PHOX2B was normalized to the amount of GUS gene transcripts (normalized $\mathrm{Ct}[\Delta \mathrm{Ct}]=$ $\mathrm{Ct}_{\mathrm{GUS}}-\mathrm{Ct}_{\mathrm{PHOX} 2 \mathrm{~B}}$ ). Samples with Ct values of PHOX2B above 40 were defined as negative.

\section{Statistical analysis}

The primary analytic endpoint was event-free survival (EFS) and overall survival (OS). Time to event was defined as the time from diagnosis until the time of the first occurrence of relapse, progression, death, or until time of the last contact if none of these occurred. Kaplan-Meier method was performed on survival analysis and the log-rank test was used to compare the survival curves between PHOX2B positive and negative subgroups. Hazard ratio (HR) and 95\% confidence interval $(95 \% \mathrm{Cl})$ were estimated

from Cox proportional hazard models. 
Statistical analyses were performed using SPSS19.0 and Graphpad Prism 5.0 software. The significance of the differences in clinical features between $\mathrm{PHOX} 2 \mathrm{~B}$ positive and negative patients was determined by the $\chi^{2}$ test. The efficacy of PHOX2B expression to diagnose metastasis was assessed by receiver operating characteristic (ROC) analysis. The P-value lower than 0.05 was considered as statistically significant.

\section{Results}

PHOX2B mRNA expression in BM and PB of patients and Patients' characteristics

A total of 276 patients, aged 1 month to 138 months (median, 35 months) with newly diagnosed neuroblastoma, were enrolled in this study. The clinical and biological characteristics were shown in Table 1. As shown in Table 2, the positive rates of the expression of PHOX2B in BM and PB of neuroblastoma patients at diagnosis were $44.6 \%$ and $35.1 \%$. The normalized $\mathrm{Ct}$ value ranged from 18.35 to 3.25 in positive BM samples, and -21.25 to 0.48 in positive PB samples, respectively. The levels of PHOX2B mRNA in BM and PB were significantly increased in patients with age $\geq 18$ months, INRGSS $M$ and MS stages, high-risk group, $L D H \geq 587 \mathrm{U} / \mathrm{L}, \mathrm{SF} \geq 92 \mathrm{ng} / \mathrm{mL}, \mathrm{NSE} \geq 100 \mathrm{ng} / \mathrm{mL}, \mathrm{MYCN}$ amplification, $1 \mathrm{p}$ loss, and BM cytology- and immunocytology-positive $(P<0.05)$. 
Table 1

Characteristics of the study patients $(n=276)$

\begin{tabular}{|llll|}
\hline Characteristics & $\mathbf{n}(\%)$ & Characteristics & $\mathbf{n}(\%)$ \\
\hline Sex & & NSE $(\mathrm{ng} / \mathrm{mL})$ & \\
\hline Male & $146(52.9)$ & $<100$ & $170(61.6)$ \\
\hline Female & $130(47.1)$ & $\geq 100$ & $106(38.4)$ \\
\hline Age, months, median, (range) & $35(1-138)$ & Bone marrow cytology & \\
\hline$<18$ months & $86(31.2)$ & Negative & $198(71.7)$ \\
\hline$\geq 18$ months & $190(68.8)$ & Positive & $78(28.3)$ \\
\hline INRGSS stage & & Bone marrow immunocytology & \\
\hline L1 & $60(21.7)$ & Negative & $184(66.7)$ \\
\hline L2 & $82(29.7)$ & Positive & $92(33.3)$ \\
\hline M & $122(44.2)$ & Pathology & \\
\hline MS & $12(4.4)$ & Neuroblastoma & $142(51.4)$ \\
\hline Risk group & & Ganglioneuroblastoma & $112(40.6)$ \\
\hline Low & $170(61.6)$ & Yes & $22(8.0)$ \\
\hline Intermediate & $106(38.4)$ & Unknown & $35(12.7)$ \\
\hline High & $60(21.7)$ & Unknown & $223(84.1)$ \\
\hline LDH (U/L) & $105(38.1)$ & MYCN & $18(6.5)$ \\
\hline$<587$ & $111(40.2)$ & No Amplification & \\
\hline$\geq 587$ & $207(75.0)$ & Unknown & \\
\hline Serum ferritin $(\mathrm{ng} / \mathrm{mL})$ & $69(25.0)$ & 1p loss & \\
\hline$<92$ & & No & \\
\hline$\geq 92$ & & Amplification & \\
\hline
\end{tabular}


Table 2

The expression of PHOX2B in newly diagnosed NB patients $(n=276)$

\begin{tabular}{|c|c|c|c|c|}
\hline \multirow[t]{2}{*}{ Characteristics } & \multicolumn{2}{|l|}{ Bone marrow } & \multicolumn{2}{|l|}{ Peripheral blood } \\
\hline & $\begin{array}{l}\text { PHOX2B } \\
\text { positive \% }\end{array}$ & $\mathbf{P}$ & PHOX2B positive \% & $\mathbf{P}$ \\
\hline All & $44.6(123 / 276)$ & & $35.1(97 / 276)$ & \\
\hline Sex & & 0.639 & & 0.151 \\
\hline Male & $45.9(67 / 146)$ & & $39.0(57 / 146)$ & \\
\hline Female & $43.1(56 / 130)$ & & $30.8(40 / 130)$ & \\
\hline Age & & 0.384 & & $<0.001$ \\
\hline$<18$ months & $40.7(35 / 86)$ & & $19.8(17 / 86)$ & \\
\hline$\geq 18$ months & $46.3(88 / 190)$ & & $42.1(80 / 190)$ & \\
\hline INRGSS stage & & $<0.001$ & & $<0.001$ \\
\hline L1 & $8.3(5 / 60)$ & & $1.7(1 / 60)$ & \\
\hline L2 & $7.3(6 / 82)$ & & $2.4(2 / 82)$ & \\
\hline M & $84.4(103 / 122)$ & & $72.1(88 / 122)$ & \\
\hline MS & $75.0(9 / 12)$ & & $50.0(6 / 12)$ & \\
\hline Risk group & & $<0.001$ & & $<0.001$ \\
\hline Low & $25.0(15 / 60)$ & & $13.3(8 / 60)$ & \\
\hline Intermediate & $16.2(17 / 105)$ & & $3.8(4 / 105)$ & \\
\hline High & $82.0(91 / 111)$ & & $76.6(85 / 111)$ & \\
\hline $\mathrm{LDH}(\mathrm{U} / \mathrm{L})$ & & $<0.001$ & & $<0.001$ \\
\hline$<587$ & $30.0(62 / 207)$ & & $18.4(38 / 207)$ & \\
\hline$\geq 587$ & $88.4(61 / 69)$ & & $85.5(59 / 69)$ & \\
\hline Serum ferritin $(\mathrm{ng} / \mathrm{mL})$ & & $<0.001$ & & $<0.001$ \\
\hline$<92$ & $21.8(37 / 170)$ & & $14.1(24 / 170)$ & \\
\hline$\geq 92$ & $81.1(86 / 106)$ & $<0.001$ & $68.9(73 / 106)$ & \\
\hline NSE (ng/mL) & & $<0.001$ & & $<0.001$ \\
\hline$<100$ & $20.0(34 / 170)$ & & $11.8(20 / 170)$ & \\
\hline
\end{tabular}




\begin{tabular}{|c|c|c|c|c|}
\hline \multirow[t]{2}{*}{ Characteristics } & \multicolumn{2}{|l|}{ Bone marrow } & \multicolumn{2}{|l|}{ Peripheral blood } \\
\hline & $\begin{array}{l}\text { PHOX2B } \\
\text { positive \% }\end{array}$ & $\mathbf{P}$ & PHOX2B positive \% & $\mathbf{P}$ \\
\hline$\geq 100$ & $84.0(89 / 106)$ & & $72.6(77 / 106)$ & \\
\hline Pathology & & 0.025 & & 0.332 \\
\hline Neuroblastoma & $47.9(68 / 142)$ & & $35.2(50 / 142)$ & \\
\hline Ganglioneuroblastoma & $33.9(38 / 112)$ & & $29.5(33 / 112)$ & \\
\hline MYCN & & $<0.001$ & & $<0.001$ \\
\hline No amplification & $40.5(94 / 232)$ & & $31.5(73 / 232)$ & \\
\hline Amplification & $73.1(19 / 26)$ & & $69.2(18 / 26)$ & \\
\hline $1 p$ loss & & $<0.001$ & & $<0.001$ \\
\hline No & $40.6(84 / 207)$ & & $30.4(63 / 207)$ & \\
\hline Yes & $80.0(28 / 35)$ & & $74.3(26 / 35)$ & \\
\hline Bone marrow cytology & & $<0.001$ & & $<0.001$ \\
\hline Negative & $23.2(46 / 198)$ & & $13.6(27 / 198)$ & \\
\hline Positive & $98.7(77 / 78)$ & & $89.7(70 / 78)$ & \\
\hline Bone marrow immunocytology & & $<0.001$ & & $<0.001$ \\
\hline Negative & $17.4(32 / 184)$ & & $8.2(15 / 184)$ & \\
\hline Positive & $98.9(91 / 92)$ & & $89.1(82 / 92)$ & \\
\hline
\end{tabular}

\section{Correlation between the expression of PHOX2B in BM and PB and metastasis}

To evaluate the ability of the PHOX2B mRNA level for predicting metastasis of neuroblastoma patients at diagnosis, ROC curves were utilized. As shown in Fig. 1A and 1B, the expressions of PHOX2B in BM and $\mathrm{PB}$ samples of neuroblastoma patients with metastatic disease were higher than patients with localized disease. The calculated area under the ROC curve was 0.906 (95\% Cl: $0.866-0.938)$ with the sensitivity and specificity of $83.6 \%$ and $93.7 \%$ at the value of -17.3 for BM (Fig. 1C), while 0.823 (95\% Cl: $0.773-$ 0.866 ) with the sensitivity and specificity of $65.7 \%$ and $97.9 \%$ at the value of -20.5 for PB (Fig. 1D). Then the calculated area under the curve of surveillance of combined PHOX2B expression in both BM and PB was 0.921 (95\% Cl: $0.883-0.950)$ for predicting metastasis of neuroblastoma at diagnosis with the sensitivity and specificity of $86.7 \%$ and $92.9 \%$, respectively (Fig. 1E). 


\section{Prognosis evaluation of the PHOX2B expression in BM and PB at diagnosis}

Among the 276 newly diagnosed patients, 30 patients were lost follow-up, and 246 patients were included in survival analysis, with a median follow-up time of 22 months. By February 15th, 2020, 38 patients had an event. The 2-year EFS was $75.7 \%$ for all patents, whereas it was $43.5 \%$ and $93.3 \%$ for patients with high-risk disease and low/intermediate-risk disease, respectively. The 2-year OS was $86.6 \%$ for all patents, whereas it was $70.6 \%$ and $95.5 \%$ for patients with high-risk disease and low/intermediate-risk disease, respectively. As shown in Fig. 2A - 2B and $3 \mathrm{~A}-3 \mathrm{~B}$, patients with the positive expression of PHOX2B in either $B M$ or $P B$ showed shorter EFS and OS than the negative patients $(P<0.001)$. Then we further analyzed the value of predicting prognostic by $\mathrm{PHOX} 2 \mathrm{~B}$ expression in patients with different risk subgroups. In the high-risk group, the patients with $\mathrm{PHOX} 2 \mathrm{~B}$ expression either in $\mathrm{BM}$ or $\mathrm{PB}$ had poorer $\mathrm{EFS}$ than those negative ones $(P<0.05$, Fig. $2 C$ and $2 D)$. Although there was no statistical difference $(P>0.05$, Fig. $2 \mathrm{C}$ and $2 \mathrm{D}$ ), the positive $\mathrm{BM}$ and $\mathrm{PB}$ patients had a poor OS trend. In the Low-/Intermediate-Risk group, $29.0 \%$ (9/31) patients with PHOX2B expression in BM had an event occurrence, while only $1.6 \%$ $(2 / 126)$ occurred in patients without PHOX2B expression $(\mathrm{P}<0.05)$. Likely, 30.0\% $(3 / 10)$ patients with PHOX2B expression in PB had event occurrence, while only 5.6\% (8/147) occurred in patients without PHOX2B expression $(\mathrm{P}<0.05)$. Unexpectedly, in Low-/Intermediate-risk group with favorable outcomes, the patients with positive expression of PHOX2B in $\mathrm{BM}$ and $\mathrm{PB}$ at diagnosis showed worse EFS and OS than the negative patients $(P<0.05$, Fig. $2 E-2 F$, Fig. $3 E-3 F)$. The univariate survival analysis showed that age $\geq 18$ months, high-risk group, MYCN amplification, chromosome 1p loss, BM and PB positive of PHOX2B, LDH $\geq 587 \mathrm{U} / \mathrm{L}, \mathrm{SF} \geq 92 \mathrm{ng} / \mathrm{mL}$, and NSE $\geq 100 \mathrm{ng} / \mathrm{mL}$ were associated with OS in patients with neuroblastoma $(P<0.05)$. Further analysis using multivariate $C O X$ proportional hazards regression model demonstrated that MYCN amplification and the positive expression of PHOX2B in BM were the independent risk factors for the prognosis of patients with neuroblastoma $(P<0.05$, Table 3$)$. 
Table 3

Univariate and multivariate analysis of clinicopathological factors for overall survival

\begin{tabular}{|lllllll|}
\hline Variables & \multicolumn{4}{l}{ Univariate } & \multicolumn{4}{l|}{ Multivariate } \\
\hline & HR & $95 \% \mathrm{Cl}$ & $\mathrm{P}$ & $\mathrm{HR}$ & $95 \% \mathrm{Cl}$ & $\mathrm{P}$ \\
\hline Risk group & 7.46 & $3.02-18.41$ & $<0.001$ & 0.41 & $0.10-1.67$ & 0.216 \\
\hline MYCN status & 9.59 & $4.51-20.37$ & $<0.001$ & 4.71 & $1.85-11.99$ & 0.001 \\
\hline 1p status & 5.58 & $2.61-11.96$ & $<0.001$ & 0.80 & $0.29-2.21$ & 0.659 \\
\hline BM PHOX2B & 20.63 & $4.89-86.98$ & $<0.001$ & 5.86 & $1.06-32.38$ & 0.043 \\
\hline PB PHOX2B & 9.35 & $3.79-23.07$ & $<0.001$ & 1.80 & $0.56-5.78$ & 0.326 \\
\hline LDH status & 10.75 & $4.72-24.78$ & $<0.001$ & 2.38 & $0.78-7.49$ & 0.138 \\
\hline Serum ferritin status & 11.43 & $3.97-32.97$ & $<0.001$ & 2.50 & $0.64-9.71$ & 0.186 \\
\hline NSE status & 8.08 & $3.26-20.01$ & $<0.001$ & 1.39 & $0.46-4.19$ & 0.556 \\
\hline
\end{tabular}

\section{Discussion}

The clinical importance of PHOX2B gene expression examination for predicting metastasis and prognostic evaluation in neuroblastoma patients were evaluated in our study. PHOX2B was first been shown as a highly sensitive and specific biomarker for neuroblastoma in $2008[8,18]$, and it was confirmed not expressed in BM and PB samples of children in the control group without neuroblastoma [8]. But in neuroblastoma patient samples, PHOX2B was detected in $32 \%$ cytology-negative and $14 \%$ immunocytology-negative BM samples, and in more than $90 \%$ of cytology-positive and immunocytologypositive BM samples [8]. Our study revealed PHOX2B was detected in 98.7\% BM cytology-positive and 98.9\% BM immunocytology-positive patients, and also in 23.2\% BM cytology-negative and $17.4 \%$ BM immunocytology-negative patients based on the high sensitivity method of RT-PCR. The positive rates of PHOX2B in BM and PB were also higher in patients with unfavorable clinically relevant factors in the present study.

Today, besides CT and MRI, ${ }^{123}$ I-MIBG and PET-CT have been widely used as more sensitive and specific imaging of neuroblastoma especially in soft tissues and bone sites $[1,19]$. However, about $10 \%$ of neuroblastomas do not take up radiolabelled MIBG [20]. Even the combined use of ${ }^{123}$ I-MIBG and PET-CT can not detect all tumor lesions in neuroblastoma patients $[19,21,22]$. So, liquid biopsy like CTCs, ctDNA, ctRNA, and even exosomes which well used in diagnosing metastasis in solid tumors need to be further studied in neuroblastoma when imaging data are ambiguous [23-26]. Neuroblastoma patients with $\geq 3$ CTCs per $4 \mathrm{ml}$ of PB were showed with an increased likelihood of having metastasis with the sensitivity and specificity of $88.89 \%$ and $78.59 \%$, respectively [27]. The univariate logistic regression results also showed that CTC and NSE levels were associated with metastasis [27]. In another study, the combined 9 
circulating miRNAs in newly diagnosed neuroblastoma patients' serum strongly associated with metastatic stage 4 disease, disease burden, and treatment response. Our data found that combined the expression of $\mathrm{PHOX} 2 \mathrm{~B}$ in both $\mathrm{BM}$ and $\mathrm{PB}$ for predicting metastasis of neuroblastoma at diagnosis with the sensitivity and specificity high to $86.7 \%$ and $92.9 \%$, respectively. That means PHOX2B is a very useful biomarker for predicting metastasis of neuroblastoma patients.

PHOX2B was also revealed to be related to the survival of neuroblastoma patients. The stage 4 neuroblastoma patients with negative expression of $\mathrm{PHOX} 2 \mathrm{~B}$ in $\mathrm{BM}$ at 3 months after diagnosis and after completion of induction chemotherapy had a favorable outcome [28]. However, the expression of PHOX2B in BM at diagnosis did not correlate to survival in patients with stage 4 neuroblastoma [28]. In another research, $19 \%$ of high-risk neuroblastoma patients at diagnosis with both high expression of PHOX2B and tyrosine hydroxylase (TH) in PB were identified as ultrahigh-risk, with 5-year EFS and OS rates of unacceptable $0 \%$ [29]. In our study, the patients with negative PHOX2B in both BM and PB at diagnosis had a more favorable prognosis than the positive patients in the high-risk group. Our data were consistent with the above studies.

The expression of PHOX2B in BM and PB in low/intermediate-risk patients were also detected in our research. Druy et al revealed the patients with positive expression of PHOX2B/TH in BM at diagnosis had a significant adverse 5-year EFS and OS in total 93 neuroblastoma patients [9]. Unfortunately, 10 patients with the expression of $\mathrm{PHOX} 2 \mathrm{~B} / \mathrm{TH}$ in $\mathrm{BM}$ at diagnosis in 69 patients with localized and stage $4 \mathrm{~S}$ neuroblastoma also had unfavorable 5-year EFS and OS. Moreover, the survival rates of these patients did not differ significantly from patients with metastatic lesions [9]. In our study, the patients with low/intermediate-risk group also had worse EFS and OS with PHOX2B positive expression in BM and PB samples at diagnosis than patients without it. The results of our research and Druy et al supported that patients with PHOX2B expression in BM or PB in the favorable group at diagnosis may suffer from disseminated disease and probably will benefit from upstaging therapy [9].

\section{Conclusion}

In conclusion, $\mathrm{PHOX} 2 \mathrm{~B}$ could be an appropriate biomarker for predicting metastasis in newly diagnosed neuroblastoma patients. The expression of PHOX2B in BM and PB at diagnosis had adverse prognostic significance in the whole group of neuroblastoma patients as well as in patients with low/intermediaterisk group.

\section{Abbreviations}

PHOX2B: Paired-like homeobox 2B; BM: Bone marrow; PB: Peripheral blood; RT-PCR: Quantitative reverse transcriptase polymerase chain reaction; ROC: Receiver operating characteristic; EFS: Event free survival; OS: Overall survival; MRD: Minimal residual disease

\section{Declarations}


Acknowledgments

The authors would like to thank the participants and study staff of the Beijing Children's Hospital, Capital Medical University, National Center for Children's Health for their contributions to this article.

\section{Authors' contributions}

CG and XM were involved in the conception and design of the study. HF and TX conceived, designed, and performed the statistical analysis and wrote the paper. $\mathrm{HH}, \mathrm{CD}$ and $\mathrm{WZ}$ participated in analyzing the data. $\mathrm{QZ}, \mathrm{XW}, \mathrm{CH}, \mathrm{YZ}, \mathrm{SZ}, \mathrm{MJ}, \mathrm{YS}$, and DZ were involved in data acquisition. All authors have read and approved the final version of the manuscript and agree to be accountable for all aspects of the work in ensuring that questions related to the accuracy or integrity of any part of the work are appropriately investigated and resolved.

\section{Funding}

This work was supported by the Capital Health Development and Scientific Research Project (Grant No: 2018-2-2095).

\section{Availability of data and materials}

The raw data are available upon reasonable request from the corresponding authors.

\section{Ethics approval and consent to participate}

The study (Clinical trial number: ChiCTR1800017940) was approved by the Ethical Committee of Beijing Children's Hospital (No. 2017-k-54). Informed written consent was obtained from each participant according to the Declaration of Helsinki.

\section{Consent for publication}

Not applicable.

\section{Competing interests}

The authors declare that they have no competing interests.

\section{References}

1. Park JR, Bagatell R, Cohn SL, Pearson AD, Villablanca JG, Berthold F, Burchill S, Boubaker A, McHugh $\mathrm{K}$, Nuchtern JG et al: Revisions to the International Neuroblastoma Response Criteria: A Consensus Statement From the National Cancer Institute Clinical Trials Planning Meeting. J Clin Onco/ 2017, 35(22):2580-2587. 
2. Pinto NR, Applebaum MA, Volchenboum SL, Matthay KK, London WB, Ambros PF, Nakagawara A, Berthold F, Schleiermacher G, Park JR et al: Advances in Risk Classification and Treatment Strategies for Neuroblastoma. J Clin Oncol 2015, 33(27):3008-3017.

3. Cheung NK, Ostrovnaya I, Kuk D, Cheung IY: Bone marrow minimal residual disease was an early response marker and a consistent independent predictor of survival after anti-GD2 immunotherapy. $J$ Clin Oncol 2015, 33(7):755-763.

4. Greaves M: Leukaemia 'firsts' in cancer research and treatment. Nat Rev Cancer 2016, 16(3):163-172.

5. Pantel K, Alix-Panabieres C: Liquid biopsy and minimal residual disease - latest advances and implications for cure. Nat Rev Clin Oncol 2019, 16(7):409-424.

6. Moss TJ, Sanders DG: Detection of neuroblastoma cells in blood. J Clin Oncol 1990, 8(4):736-740.

7. Pattyn A, Morin X, Cremer H, Goridis C, Brunet JF: The homeobox gene Phox2b is essential for the development of autonomic neural crest derivatives. Nature 1999, 399(6734):366-370.

8. Stutterheim J, Gerritsen A, Zappeij-Kannegieter L, Kleijn I, Dee R, Hooft L, van Noesel MM, Bierings M, Berthold F, Versteeg $\mathrm{R}$ et al: PHOX2B is a novel and specific marker for minimal residual disease testing in neuroblastoma. J Clin Oncol 2008, 26(33):5443-5449.

9. Druy AE, Shorikov EV, Tsaur GA, Popov AM, Zaychikov AN, Tuponogov SN, Saveliev LI, Tytgat GAM, Fechina LG: Prospective investigation of applicability and the prognostic significance of bone marrow involvement in patients with neuroblastoma detected by quantitative reverse transcription PCR. Pediatr Blood Cancer 2018, 65(11):e27354.

10. Tchirkov A, Greze V, Plantaz D, Rouel N, Vago P, Kanold J: Very long-term molecular follow-up of minimal residual disease in patients with neuroblastoma. Pediatr Blood Cancer 2018, 65(12):e27404.

11. van Wezel EM, Stutterheim J, Vree F, Zappeij-Kannegieter L, Decarolis B, Hero B, Berthold F, Schumacher-Kuckelkorn R, Simon T, Fiocco M et al: Minimal residual disease detection in autologous stem cell grafts from patients with high risk neuroblastoma. Pediatr Blood Cancer 2015, 62(8):13681373.

12. Marachelian A, Villablanca JG, Liu CW, Liu B, Goodarzian F, Lai HA, Shimada H, Tran HC, Parra JA, Gallego R et al: Expression of Five Neuroblastoma Genes in Bone Marrow or Blood of Patients with Relapsed/Refractory Neuroblastoma Provides a New Biomarker for Disease and Prognosis. Clin Cancer Res 2017, 23(18):5374-5383.

13. Monclair T, Brodeur GM, Ambros PF, Brisse HJ, Cecchetto G, Holmes K, Kaneko M, London WB, Matthay KK, Nuchtern JG et al: The International Neuroblastoma Risk Group (INRG) staging system: an INRG Task Force report. J Clin Oncol 2009, 27(2):298-303.

14. Cohn SL, Pearson AD, London WB, Monclair T, Ambros PF, Brodeur GM, Faldum A, Hero B, lehara T, Machin D et al: The International Neuroblastoma Risk Group (INRG) classification system: an INRG Task Force report. J Clin Oncol 2009, 27(2):289-297.

15. Wang X, Wang L, Su Y, Yue Z, Xing T, Zhao W, Zhao Q, Duan C, Huang C, Zhang D et al: Plasma cellfree DNA quantification is highly correlated to tumor burden in children with neuroblastoma. Cancer Med 2018. 
16. Su Y, Wang L, Wang X, Yue Z, Xing T, Zhao W, Zhao Q, Duan C, Huang C, Han Y et al: Dynamic alterations of plasma cell free DNA in response to chemotherapy in children with neuroblastoma. Cancer Med 2019, 8(4):1558-1566.

17. Li W, Cui L, Gao C, Liu S, Zhao X, Zhang R, Zheng H, Wu M, Li Z: DNMT3A mutations in Chinese childhood acute myeloid leukemia. Medicine (Baltimore) 2017, 96(31):e7620.

18. Viprey VF, Lastowska MA, Corrias MV, Swerts K, Jackson MS, Burchill SA: Minimal disease monitoring by QRT-PCR: guidelines for identification and systematic validation of molecular markers prior to evaluation in prospective clinical trials. J Pathol 2008, 216(2):245-252.

19. Taggart DR, Han MM, Quach A, Groshen S, Ye W, Villablanca JG, Jackson HA, Mari Aparici C, Carlson $\mathrm{D}$, Maris $\mathrm{J}$ et al: Comparison of iodine-123 metaiodobenzylguanidine (MIBG) scan and [18F]fluorodeoxyglucose positron emission tomography to evaluate response after iodine-131 MIBG therapy for relapsed neuroblastoma. J Clin Oncol 2009, 27(32):5343-5349.

20. Shulkin BL, Shapiro B: Current concepts on the diagnostic use of MIBG in children. J Nucl Med 1998, 39(4):679-688.

21. Melzer HI, Coppenrath E, Schmid I, Albert MH, von Schweinitz D, Tudball C, Bartenstein P, Pfluger T: (1)(2)(3)I-MIBG scintigraphy/SPECT versus (1)(8)F-FDG PET in paediatric neuroblastoma. Eur J NuCl Med Mol Imaging 2011, 38(9):1648-1658.

22. Sharp SE, Shulkin BL, Gelfand MJ, Salisbury S, Furman WL: 123I-MIBG scintigraphy and 18F-FDG PET in neuroblastoma. $J$ Nucl Med 2009, 50(8):1237-1243.

23. Siravegna G, Marsoni S, Siena S, Bardelli A: Integrating liquid biopsies into the management of cancer. Nat Rev Clin Oncol 2017, 14(9):531-548.

24. Luskin MR, Murakami MA, Manalis SR, Weinstock DM: Targeting minimal residual disease: a path to cure? Nat Rev Cancer 2018, 18(4):255-263.

25. De Mattos-Arruda L, Cortes J, Santarpia L, Vivancos A, Tabernero J, Reis-Filho JS, Seoane J: Circulating tumour cells and cell-free DNA as tools for managing breast cancer. Nat Rev Clin Oncol 2013, 10(7):377-389.

26. Crowley E, Di Nicolantonio F, Loupakis F, Bardelli A: Liquid biopsy: monitoring cancer-genetics in the blood. Nat Rev Clin Oncol 2013, 10(8):472-484.

27. Liu X, Zhang Z, Zhang B, Zheng Y, Zheng C, Liu B, Zheng S, Dong K, Dong R: Circulating tumor cells detection in neuroblastoma patients by EpCAM-independent enrichment and immunostainingfluorescence in situ hybridization. EBioMedicine 2018, 35:244-250.

28. Stutterheim J, Zappeij-Kannegieter L, Versteeg R, Caron HN, van der Schoot CE, Tytgat GA: The prognostic value of fast molecular response of marrow disease in patients aged over 1 year with stage 4 neuroblastoma. Eur J Cancer 2011, 47(8):1193-1202.

29. Viprey VF, Gregory WM, Corrias MV, Tchirkov A, Swerts K, Vicha A, Dallorso S, Brock P, Luksch R, Valteau-Couanet $D$ et al: Neuroblastoma mRNAs predict outcome in children with stage 4 neuroblastoma: a European HR-NBL1/SIOPEN study. J Clin Oncol 2014, 32(10):1074-1083. 
A

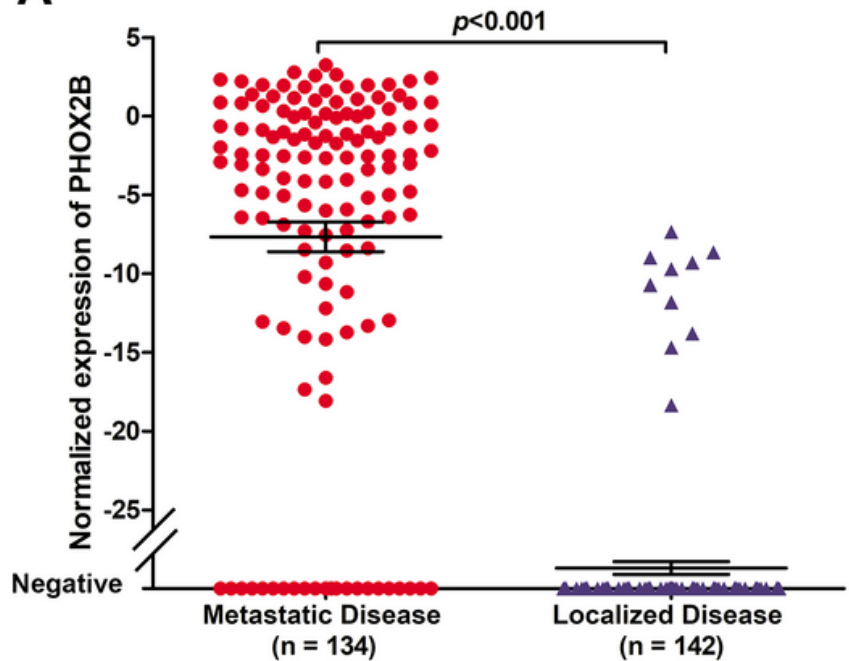

C

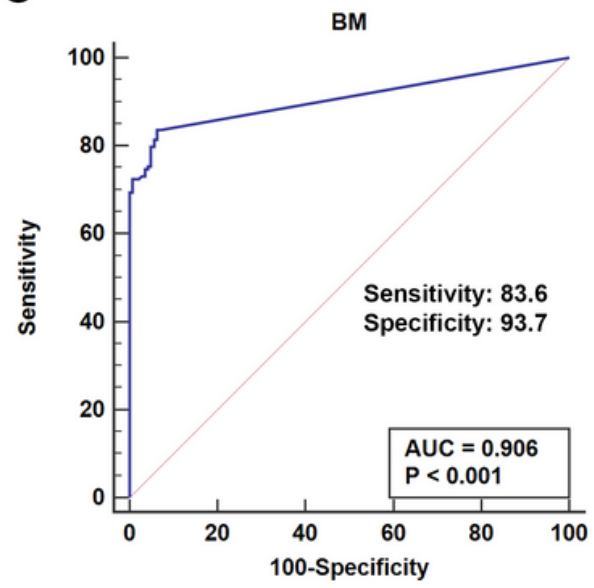

B

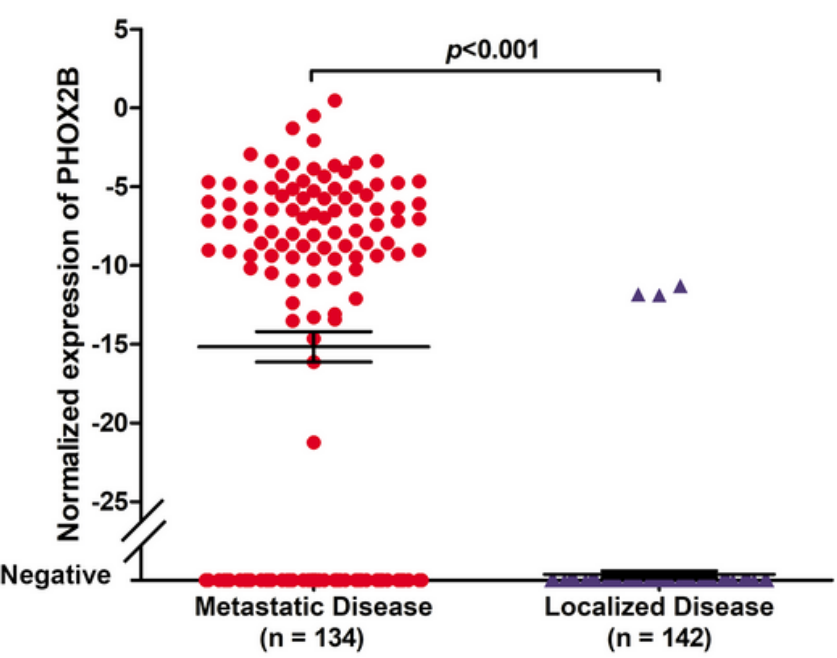

PB

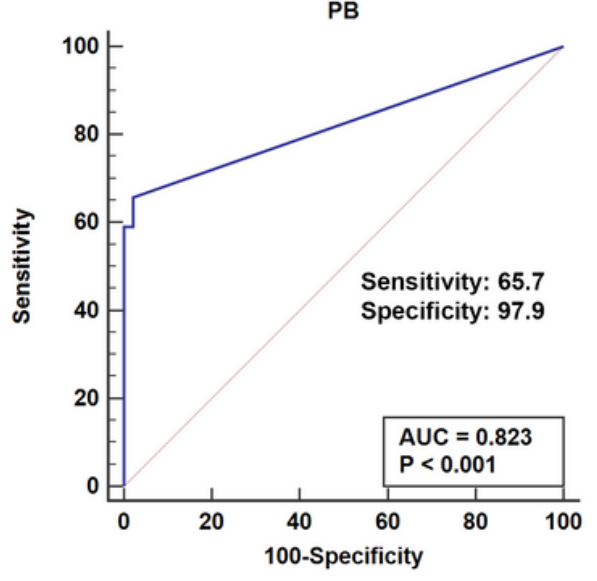

E

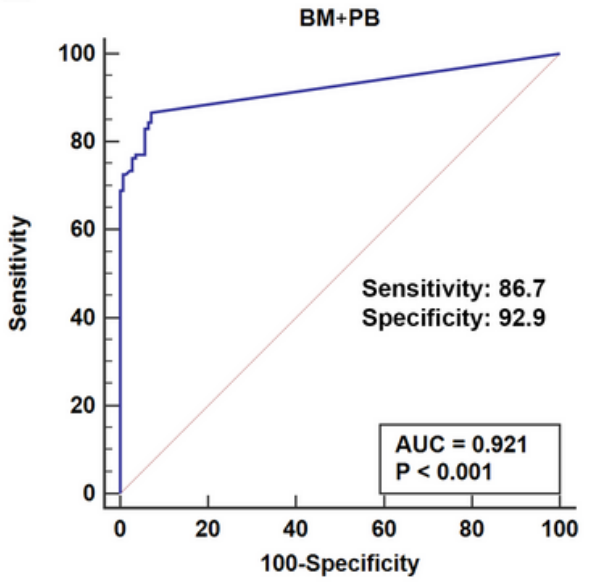

\section{Figure 1}

The expression of $\mathrm{PHOX} 2 \mathrm{~B}$ in $\mathrm{BM}$ and $\mathrm{PB}$ samples for predicting metastasis of neuroblastoma. The expression of $\mathrm{PHOX} 2 \mathrm{~B}$ in (A) $\mathrm{BM}$, and in (B) PB of neuroblastoma patient with metastatic disease and localized disease. The ROC curve of the expression of PHOX2B in (C) BM, and (D) PB, and (E) the combined of $\mathrm{PHOX} 2 \mathrm{~B}$ in $\mathrm{BM}$ and $\mathrm{PB}$ for discriminating patients with and without metastasis. 
A
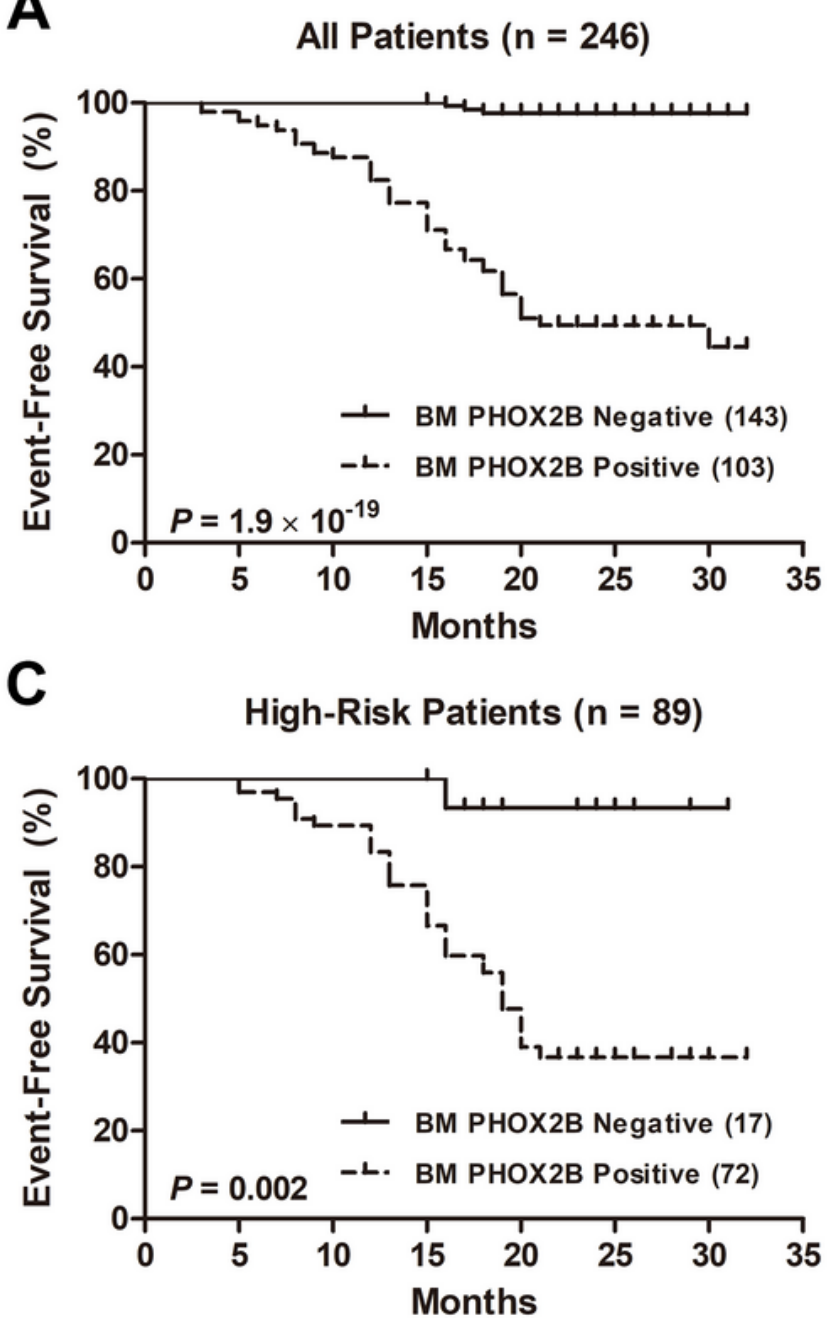

E Low/Intermediate-Risk Patients ( $n=157)$

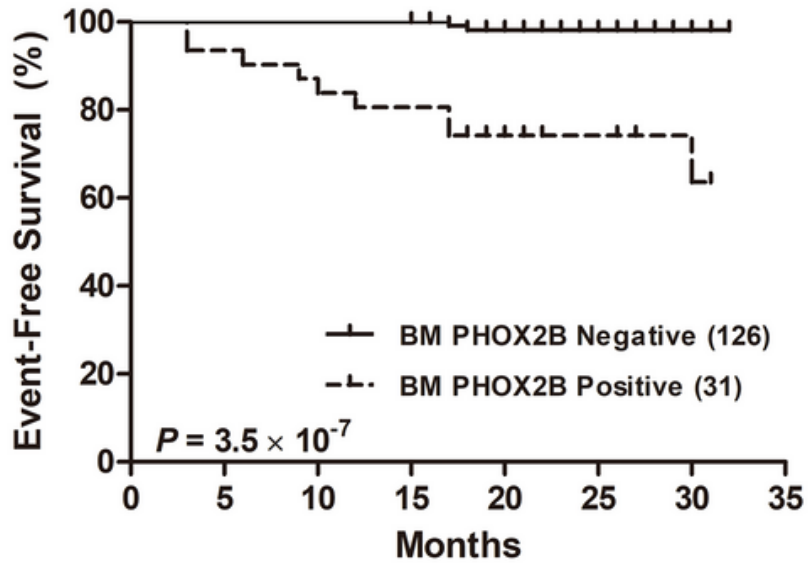

B

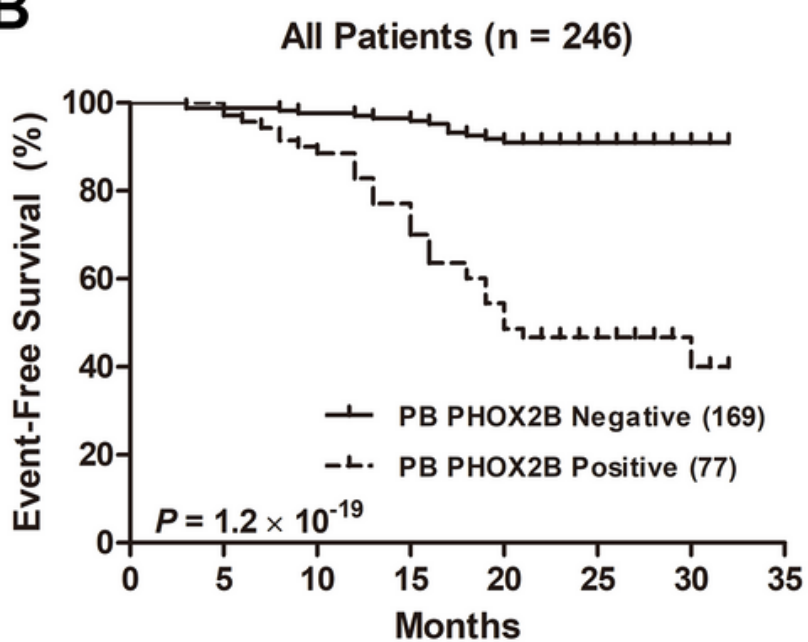

Digh-Risk Patients $(n=89)$

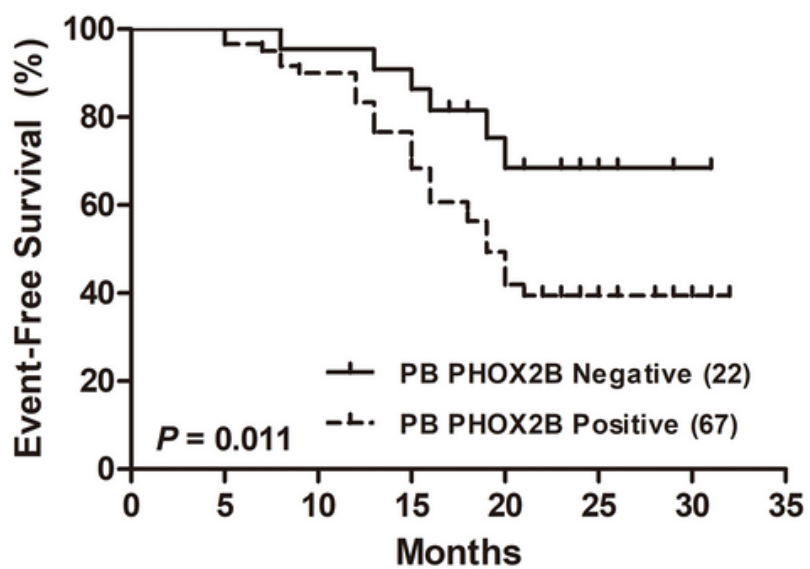

F Low/Intermediate-Risk Patients ( $n=157$ )

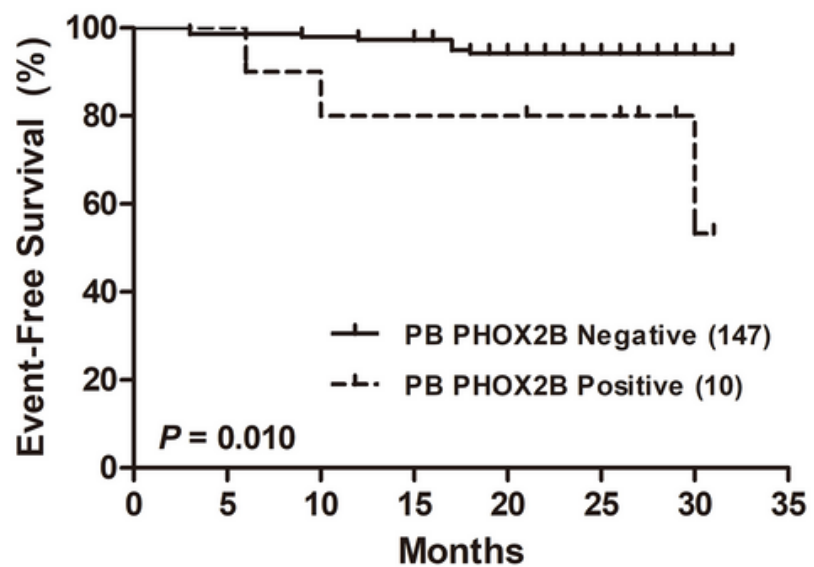

Figure 2

Event-free survival of positive and negative expression of PHOX2B in BM and PB of neuroblastoma patients. EFS of different expression of PHOX2B in BM of (A) all neuroblastoma patients, (C) high-risk patients, and (E) low-/intermediate-risk group. EFS of different expression of PHOX2B in PB of (B) all neuroblastoma patients, (D) high-risk patients, and (F) low-/intermediate-risk group. 
A

All Patients $(n=246)$

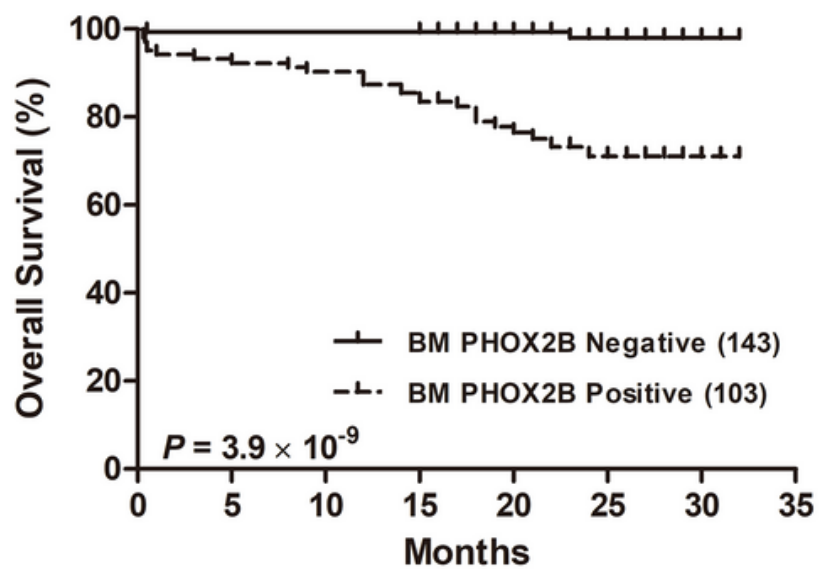

C High-Risk Patients $(\mathrm{n}=89)$

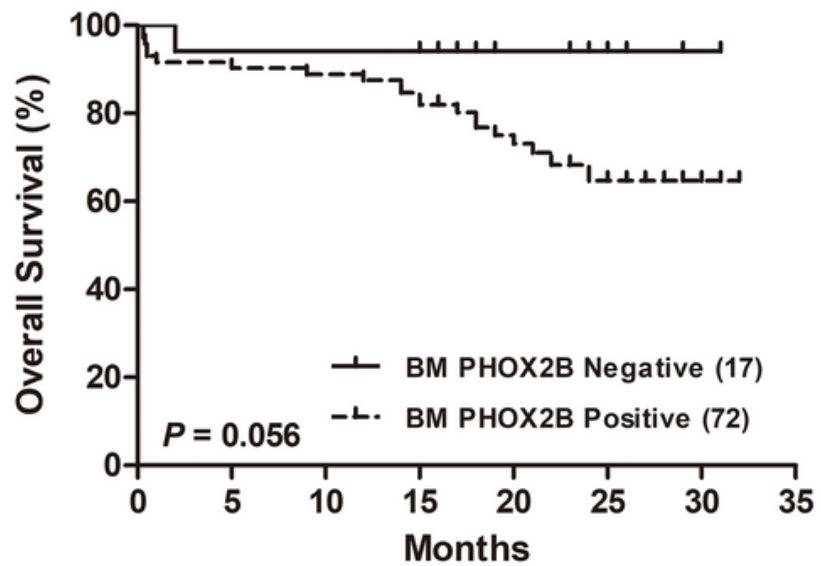

E Low/Intermediate-Risk Patients $(n=157)$

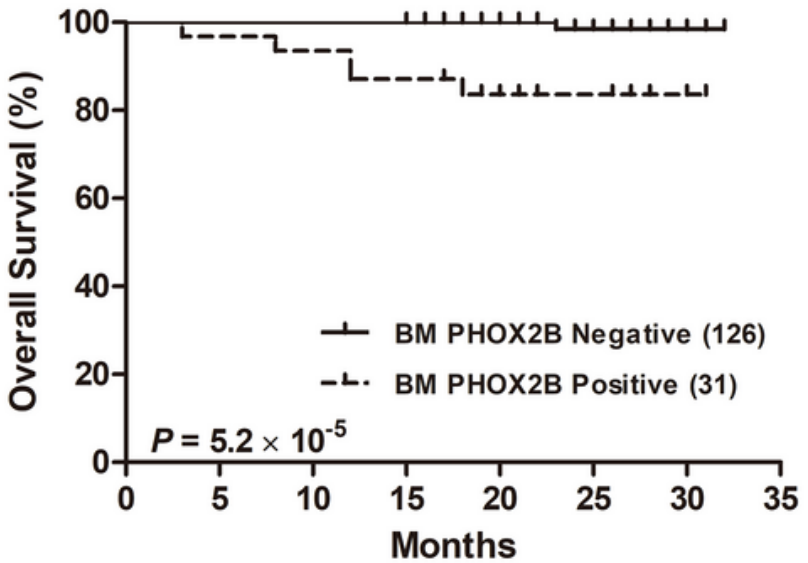

B All Patients $(n=246)$

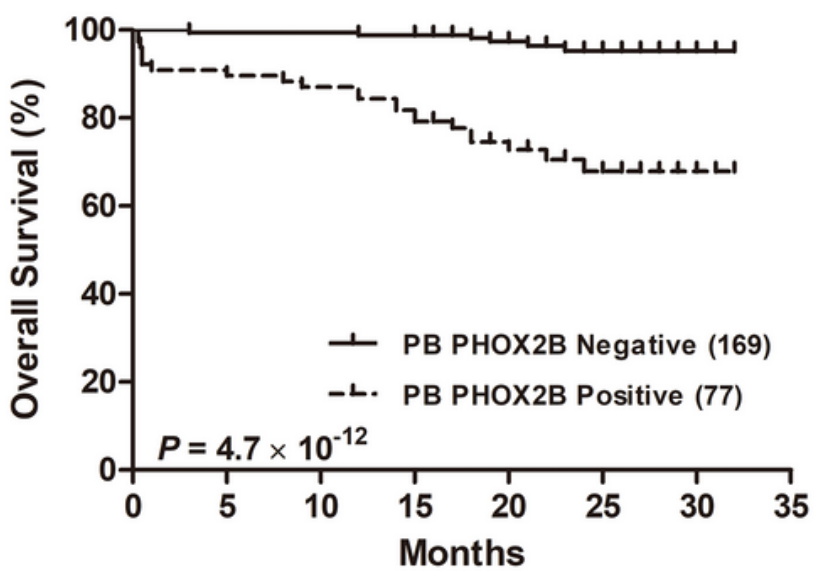

D High-Risk Patients $(n=89)$

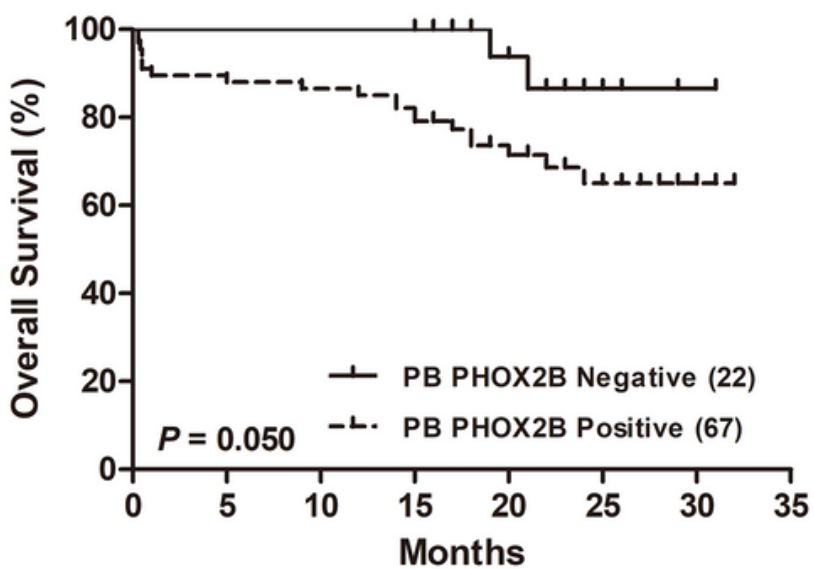

$\mathbf{F}$

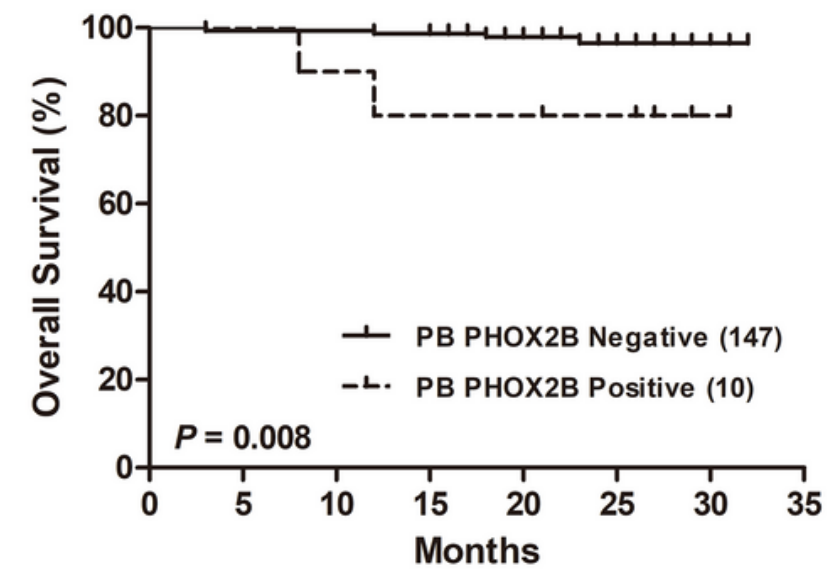

Figure 3

Overall survival of positive and negative expression of PHOX2B in BM and PB of neuroblastoma patients. OS of different expression of PHOX2B in BM of (A) all neuroblastoma patients, (C) high-risk patients, and (E) low-/intermediate-risk group. OS of different expression of PHOX2B in PB of (B) all neuroblastoma patients, (D) high-risk patients, and (F) low-/intermediate-risk group. 